\title{
La Reserva de la Biosfera Meseta Ibérica como espacio natural protegido transfronterizo: ¿herramienta de conservación o marca promocional?
}

\author{
Juan Manuel Trillo Santamaría \\ Valerià Paül Carril \\ Universidade de Santiago de Compostela. Departamento de Xeografía \\ juanmanuel.trillo@usc.es \\ v.paul.carril@usc.es
}

Recibido: enero de 2018

Aceptado: mayo de 2018

Publicado: julio 2018

\section{Resumen}

Los espacios naturales protegidos transfronterizos (ENPT) se han multiplicado en los últimos años, sobre todo en el ámbito europeo y en África Austral. Tanto desde las organizaciones internacionales como desde el mundo académico se han realizado multitud de investigaciones acerca de los ENPT; más neutras, por lo general, las primeras, y más críticas las segundas. En la Península Ibérica, destaca por su extensión la Reserva de la Biosfera Transfronteriza Meseta Ibérica, declarada en 2015. Este artículo la estudia a través de una investigación cualitativa, en particular mediante entrevistas semiestructuradas. Los resultados obtenidos son interpretados en el marco de las referencias disponibles sobre los ENPT. El análisis efectuado revela que esta Reserva de la Biosfera se basa discursivamente más en cuestiones turísticas y de marca que en aspectos vinculados a la protección y conservación ambientales, una conclusión que subvierte el modelo esperado.

Palabras clave: espacio natural protegido transfronterizo (ENPT); reserva de la biosfera; turismo; conservación ambiental; España/Portugal

Resum. La Reserva de la Biosfera Meseta Ibérica com a espai natural protegit transfronterer: eina de conservació o marca promocional?

Els espais naturals protegits transfronterers (ENPT) s'han multiplicat durant els darrers anys, sobretot a l'àmbit europeu i a l'Àfrica Austral. Tant des de les organitzacions internacionals com des del món acadèmic s'han fet multitud de recerques quant als ENPT; més neutres, en general, les primeres, i més crítiques les segones. A la península Ibèrica, hi destaca per la seva extensió la Reserva de la Biosfera Transfronterera Meseta Ibérica, declarada 
el 2015. Aquest article l'estudia a través d'una recerca qualitativa, en particular mitjançant entrevistes semiestructurades. Els resultats són interpretats en el marc de les referències disponibles sobre els ENPT. L'anàlisi feta revela que aquesta reserva de la biosfera es basa discursivament més en qüestions turístiques i de marca que no pas en aspectes vinculats a la protecció i la conservació ambientals, una conclusió que subverteix el model esperat.

Paraules clau: espai natural protegit transfronterer (ENPT); reserva de la biosfera; turisme; conservació ambiental; Espanya/Portugal

Résumé. La réserve de biosphère Meseta ibérica en tant qu'espace naturel protégé transfrontalier: outil de conservation ou marque promotionnelle?

Les espaces naturels protégés transfrontaliers (ENPTs) se sont multipliés au cours des dernières années, en particulier dans le contexte européen et de l'Afrique australe. Tant depuis les organisations internationales que le monde académique des nombreuses études sur les ENPTs ont été réalisées ; plus neutres, en général, les premières, et plus critiques les secondes. Dans la Péninsule Ibérique, la réserve de biosphère transfrontalière Meseta ibérica, déclarée en 2015, est remarquable par sa dimension. Cet article l'étudie à travers une recherche qualitative, en particulière, par des entretiens semi-directifs. Les résultats obtenus sont interprétés dans le cadre des références disponibles sur les ENPTs. L'analyse effectuée révèle que cette réserve de biosphère est plus basée dans son discours sur des questions touristiques et de marque que sur des aspects liés à la protection et à la conservation de l'environnement, conclusion bouleversant le modèle initialement prévu.

Mots-clés: espace naturel protégé transfrontalier (ENPT); réserve de biosphère; tourisme; conservation de l'environnement; Espagne/Portugal

\section{Abstract. The Meseta Ibérica Transboundary Biosphere Reserve as a transboundary protected natural space: a conservation tool or a promotional brand?}

Transboundary protected areas (TBPAs) have multiplied in recent years, especially in Europe and Southern Africa. A vast body of research has been carried out on TBPAs by international organisations as well as scholars, with the former generally being more neutral and the latter more critical. In the Iberian Peninsula, the Meseta Ibérica Transboundary Biosphere Reserve, designated in 2015, stands out for its covered land area. Qualitative research methods are used to study this biosphere reserve, particularly semi-structured interviews. The results are interpreted within the framework of the available literature on TBPAs. The analysis reveals that this biosphere reserve is discursively based more on tourism and brand issues than on aspects related to environmental protection and conservation; a conclusion which subverts the expected model.

Keywords: transboundary protected area (TBPA); biosphere reserve; tourism; environmental conservation; Spain/Portugal

\section{Sumario}

\section{Introducción}

2. Marco teórico

3. La Reserva de la Biosfera Transfronteriza Meseta Ibérica
4. Notas metodológicas

5. Resultados

6. Discusión y conclusiones

Referencias bibliográficas 


\section{Introducción}

En un espacio de una gran densidad y tradición de cooperación como son las regiones fronterizas del noroeste peninsular (López Trigal et al., 1995; López Trigal y Guichard, 2000; López Trigal, 2017; Paül et al., 2017), encontramos una iniciativa reciente que destaca por diversos motivos: la Reserva de la Biosfera Transfronteriza Meseta Ibérica. En primer lugar, es fruto de un proyecto INTERREG, que financia la candidatura para su presentación ante la UNESCO. En segundo lugar, se utiliza la figura jurídica comunitaria de la Agrupación Europea de Cooperación Territorial (AECT) para gestionar la reserva, en concreto, ZASNET. En tercer lugar, se trata de un proceso relativamente rápido, si tenemos en cuenta los largos periodos de tiempo que pueden pasar entre una propuesta de candidatura y la declaración por parte de la UNESCO. En cuarto lugar, estamos ante un caso poco habitual, ya que, según datos de la propia UNESCO, solo existen 20 reservas de la biosfera transfronterizas, de un total de $699^{1}$. Y, por último, esta Reserva presenta unas dimensiones de gran tamaño, que la convierten en la mayor de la Península Ibérica. Por todo lo anterior, creemos que tiene sentido reflexionar sobre ella en relación con el cuerpo teórico desarrollado acerca de los espacios naturales protegidos transfronterizos (ENPT). De este modo, buscamos observar si los procesos que llevan a establecer esta figura siguen las pautas de gobernanza que se señalan en la teoría. No pretendemos elaborar un estudio de las funcionalidades ecológicas o sociopolíticas de la Reserva, sino que perseguimos encontrar las voces que nos hablen de las razones y los pasos dados para alcanzar un dispositivo transfronterizo.

El presente texto comienza con un marco teórico en torno a la consecución de un modelo analítico para estudiar los ENPT; se ha de recordar que una reserva de la biosfera es un ENP de acuerdo con la bibliografía de referencia (Depraz, 2008) y, en particular para el caso de España, según el artículo 50 —en su redacción actualizada - de la Ley 42/2007, que la declara como uno de los tipos de «área protegida por instrumento internacional». A continuación, se presenta el ámbito de estudio, para proseguir con unas consideraciones metodológicas, en las que se apuesta por el uso de las entrevistas semiestructuradas. La exposición de los resultados, el siguiente apartado, da voz a las personas entrevistadas. El texto se cierra con una discusión y conclusiones finales que retoman los elementos teóricos para interpretar los resultados.

\section{Marco teórico}

A partir de Sandwith et al. (2001), trabajo que actúa como punta de lanza en materia de ENPT, se han multiplicado las aportaciones que tratan sobre estos espacios protegidos. Algunas presentan una continuidad obvia, ya sea por su

1. <http://www.unesco.org/new/en/natural-sciences/environment/ecological-sciences/biosphere-reserves/transboundary-biosphere-reserves/> [consulta: 16 de diciembre de 2017]. 
autoría o bien por el empleo de la misma perspectiva —ofrecer una guía desde una organización internacional para que pueda ser utilizada en múltiples contextos-, por ejemplo, Braack et al. (2006), Sandwith y Lockwood (2006), EUROPARC Federation (2010) o Erg et al. (2012). Otras, en cambio, han adoptado un enfoque más distanciado y menos aséptico, sobre todo desde el ámbito académico, que permite poner en tela de juicio parte de las referencias «oficiales»; es el caso de Lange (2009), Büscher (2010), Leibenath et al. (2010) o Trillo y Paül (2016). A continuación, el presente apartado ofrece un modelo analítico para los ENPT sobre la base de los conceptos manejados en esta bibliografía disponible, a pesar de que autores como McKinney y Vasilijević (2012) hayan señalado la carencia de un referente único para estos dispositivos.

En primer lugar, se detecta la coincidencia en apuntar unas bases de partida necesarias para poder llegar a desarrollar un ENPT dado, a saber:

- Debe existir un acuerdo respecto de los valores compartidos y una visión común que permitan superar la frontera. Por ejemplo, ha de identificarse una motivación o un vector (una especie o especies, un paisaje o paisajes, etc.) que impulsen la iniciativa. Esa visión conjunta se ha definido como una orientación futura acordada por los actores participantes (Lange, 2009; EUROPARC Federation, 2010; McKinney y Vasilijević, 2012). Resulta también clave que se produzca un compromiso alrededor de estos valores y esta visión por parte del nivel decisor, normalmente político-administrativo.

- Organización de actividades de colaboración entre los parques o espacios protegidos implicados, que involucren sobre todo a quienes los gestionan. En este sentido, la nómina de posibilidades es larga y abarca desde reuniones periódicas y constitución de equipos transnacionales hasta cursos de capacitación lingüística para mejorar la comunicación, trabajo de campo conjunto o desarrollo de un SIG común y compartir instrumentos de trabajo.

- Lo expuesto en los anteriores puntos debe concernir no solo a las personas que tengan responsabilidades políticas o de gestión, sino también a la sociedad civil y la población local (Braack et al., 2006; Leibenath et al., 2010; Erg et al., 2012). En particular, resultan relevantes los grupos directamente afectados (residentes, empresarios con negocios en la zona, etc.) y aquellos colectivos más implicados en aspectos ambientales (ecologistas y naturalistas, voluntariado ambiental, etc.).

En segundo lugar, la bibliografía concede una notable importancia a la existencia de un documento de planificación, estratégico o no; difiere, no obstante, en cuál debe ser su alcance, pues puede contener aspectos tales como normativa, conservación, desarrollo económico, turismo, bienestar social, infraestructuras, seguridad y/o acciones concretas, dependiendo de los distintos autores (Sandwith et al., 2001; Braack et al., 2006; Lange, 2009; McKinney y Vasilijević, 2012). Algunos de ellos recomiendan que, antes del plan propiamente dicho, se firme un acuerdo escrito de colaboración entre las 
partes. Como siempre que se produce planificación, se debe estar atento a la implantación, ya que es posible que exista un desajuste entre lo planificado y lo ejecutado. Para ello resulta necesario monitorizar el desarrollo del plan (Sandwith et al., 2001; McKinney y Vasilijević, 2012).

En tercer lugar, algunos autores indican que un ENPT solo se consolidará si se llega a crear una estructura organizativa conjunta que supere la frontera (Büscher y Schoon, 2009; Oszlanyi, 2001). Ello ha sido propuesto en el caso concreto de las reservas de la biosfera transfronterizas (Trillo y Paül, 2016), para las que incluso se ha diseñado la arquitectura institucional necesaria: secretariado permanente, reuniones conjuntas regulares, etc. En el contexto de la Unión Europea, las AECT parecen una solución operativa en este sentido (Paül et al., 2017); de hecho, ya existen algunos casos, por ejemplo, Alpi MarittimeMercantour y Bouches de Bonifacio (ambas en funcionamiento desde 2013 y localizadas entre Francia e Italia).

Finalmente, en la revisión de la bibliografía se detecta cierta obsesión por la cuestión financiera, de modo que se insiste en la consecución de fondos o atracción de inversiones a medida que el ENPT se va desarrollando (Sandwith et al., 2001; EUROPARC Federation, 2010; Erg et al., 2010). No obstante, algunos autores como Lange (2009), Büscher (2010), Leibenath et al. (2010) o Trillo y Paül (2016) han sido críticos de un modo u otro con tal fijación, hasta el punto de que han sugerido que en contextos como el europeo o el de África Austral la cooperación entre parques se produce, sobre todo, porque se pretende acceder a fondos e, incluso, finaliza una vez que remiten los mismos.

\section{La Reserva de la Biosfera Transfronteriza Meseta Ibérica}

Se trata de la reserva de la biosfera más grande de la Península Ibérica, con más de un millón de hectáreas, el $58 \%$ de las cuales corresponde al territorio portugués y el $42 \%$ restante, al español. En Portugal, engloba un total de 12 municipios pertenecientes al distrito de Bragança (todos excepto Torre de Moncorvo), más Figueira de Castelo Rodrigo (distrito da Guarda). En España, está conformada por una franja de Castilla y León constituida por 75 municipios que siguen la frontera desde el límite con Galicia hasta las inmediaciones de la extremeña: en Zamora, recoge buena parte o todo el ámbito de las comarcas de Sanabria (incluyendo As Portelas o Alta Sanabria), Carballeda, Aliste y Sayago, con la totalidad de la Sierra de la Culebra; en Salamanca, incluye en lo fundamental el ámbito del Parque Natural de Arribes del Duero y su área de influencia, hasta Villar de la Yegua al sur $(15 \mathrm{~km}$ al norte de Ciudad Rodrigo) (figura 1).

De acuerdo con la información disponible, la Reserva de la Biosfera fue promovida por la AECT ZASNET ${ }^{2}$ con el objetivo de ser «la mayor reserva

2. ZASNET está constituida por dos asociaciones de municipios portuguesas (Terra Fria do Nordeste Transmontano y Terra Quente Transmontana), la Câmara Municipal de 
Figura 1. La Reserva de la Biosfera Transfronteriza Meseta Ibérica

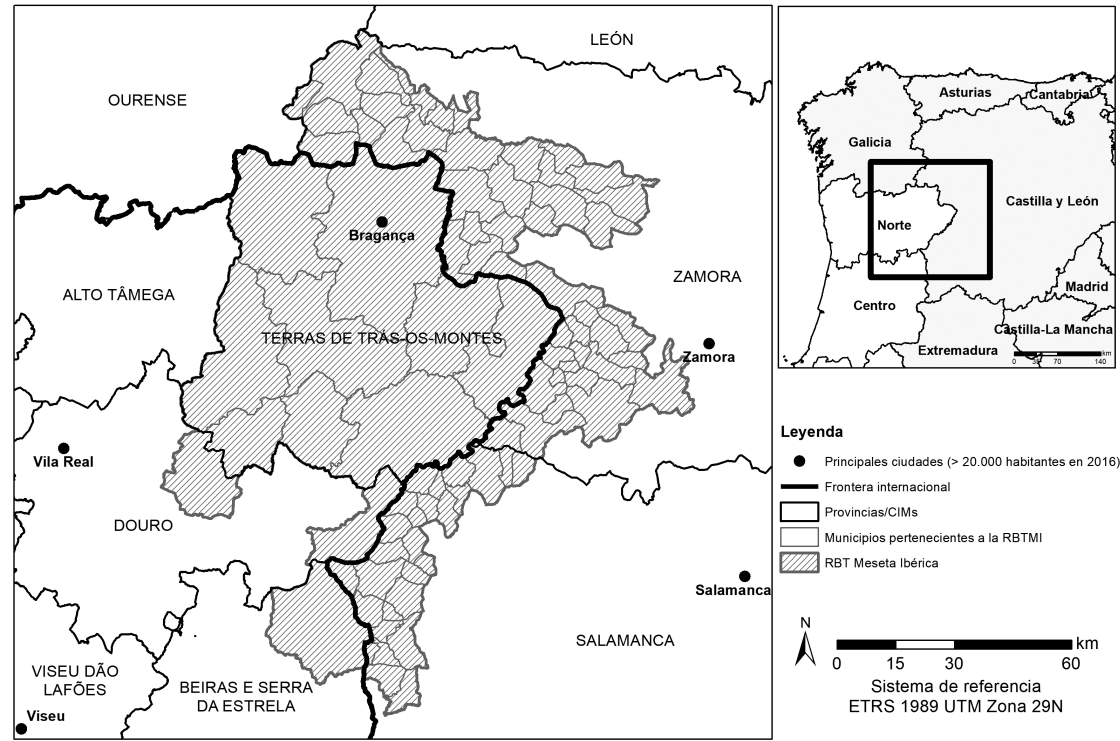

Fuente: elaboración de Alejandro Gómez Pazo. Base cartográfica del @Instituto Geográfico Nacional.

de la biosfera transfronteriza de Europa $»^{3}$. Declarada así por la UNESCO en 2015, presenta, como toda reserva de la biosfera, una zonificación tripartita: núcleo, tampón y transición. Las dos primeras se corresponden en términos generales con espacios ya protegidos: los parques naturales de Arribes del Duero, Douro Internacional — ambos contiguos a lado y lado de la frontera-, Lago de Sanabria ${ }^{4}$ y Montesinho —estos dos últimos sin contigüidad física-. Cabe tener en cuenta que los parques naturales castellano-leoneses y los portugueses están gestionados de modo distinto, de forma que los segundos no

Bragança, las diputaciones de Salamanca y Zamora y el Ayuntamiento de Zamora. Con sede en Bragança, fue aprobada en 2010 al publicarse sus estatutos en el Diário da República. De acuerdo con su página web, «Una de las ventajas principales de esta forma de organización es desarrollar estrategias conjuntas para captar fondos comunitarios"; entre sus proyectos desarrollados, destaca claramente la Reserva de la Biosfera. Véase <http://www.zasnet-aect. eu/> [consulta: 5 de noviembre de 2017]. Así, su ámbito territorial reúne las provincias de Salamanca y Zamora, más la mayor parte del distrito de Bragança (los distritos en Portugal no tienen una administración asociada), pues las dos asociaciones de municipios lo cubren en su práctica totalidad (10 de 12 municipios, excluidos Freixo de Espada à Cinta y Torre de Moncorvo).

3. <http://www.biosfera-mesetaiberica.com/es/es/candidatura> [consulta: 5 de noviembre de 2017].

4. Llamado de «Lago de Sanabria y alrededores» hasta 2017, en la actualidad ha cambiado su nombre por "Lago de Sanabria y Sierras Segunde[i] ra y de Porto» (Ley 1/2017 de Castilla y León). 
Figura 2. Típico paisaje del zócalo meseteño, en las inmediaciones de Villalcampo (Zamora), dentro del Parque Natural Arribes del Duero: ondulado, pedregoso, con tierras cercadas por muros y muchos campos abandonados

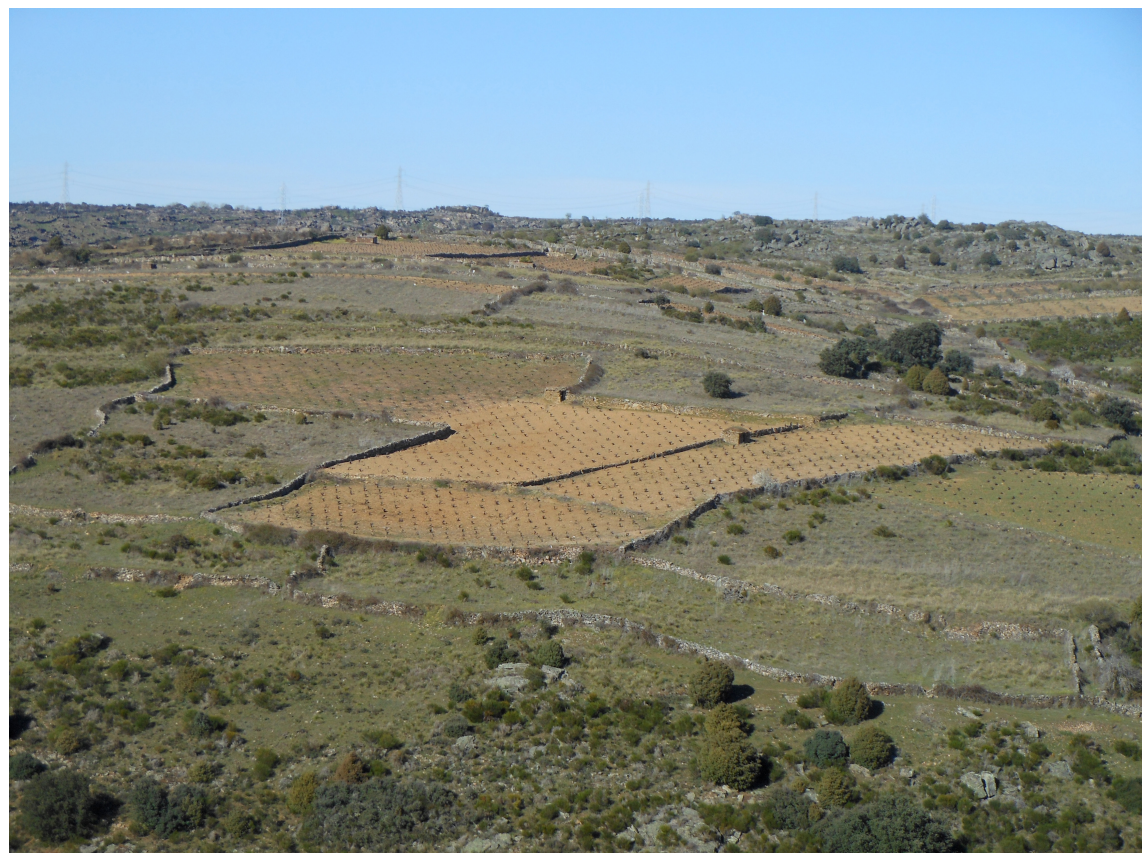

Fuente: fotografía de Valerià Paül (30 de marzo de 2015).

cuentan con una estructura estable de administración y conservación in situ. A estos cuatro parques naturales cabe sumar parte del Parque Natural Regional do Vale do Tua, gestionado por una agencia de desarrollo regional, y la totalidad de la Reserva Regional de Caza/Lugar de Interés Comunitario (LIC) (desde 2015, Zona Especial de Conservación, ZEC) de la Sierra de la Culebra.

De acuerdo con la publicación oficial en el BOE, la Reserva «incluye una gran variedad de paisajes con una elevada riqueza de elementos orográficos, y una heterogeneidad marcada en la ocupación del suelo, que en conjunto constituye una matriz riquísima de elementos paisajísticos» (27/10/2015: 76366), un descriptor que podría ser aplicado a prácticamente cualquier lugar. Por la denominación, se entiende que su vector principal es la presencia del retazo de afloramiento del Macizo Hespérico —el zócalo de origen varisco, granítico y arrasado - en el que se alternan plataformas más o menos onduladas con profundas incisiones fluviales. Constituye un paisaje contrastado de penillanuras y tajos característico de buena parte, aunque no de toda, de la superficie incluida en la Reserva de la Biosfera. El moldeado humano de este paisaje es intenso, peculiar e impresionante, aunque se encuentra en vías de desaparición (Prada, 2014). 
Figura 3. El Duero/Douro desde Miranda do Douro (Trás-os-Montes), hoy embalsado, encajado en un tajo de unos $200 \mathrm{~m}$ de caída, que en otros puntos supera los $300 \mathrm{~m}$

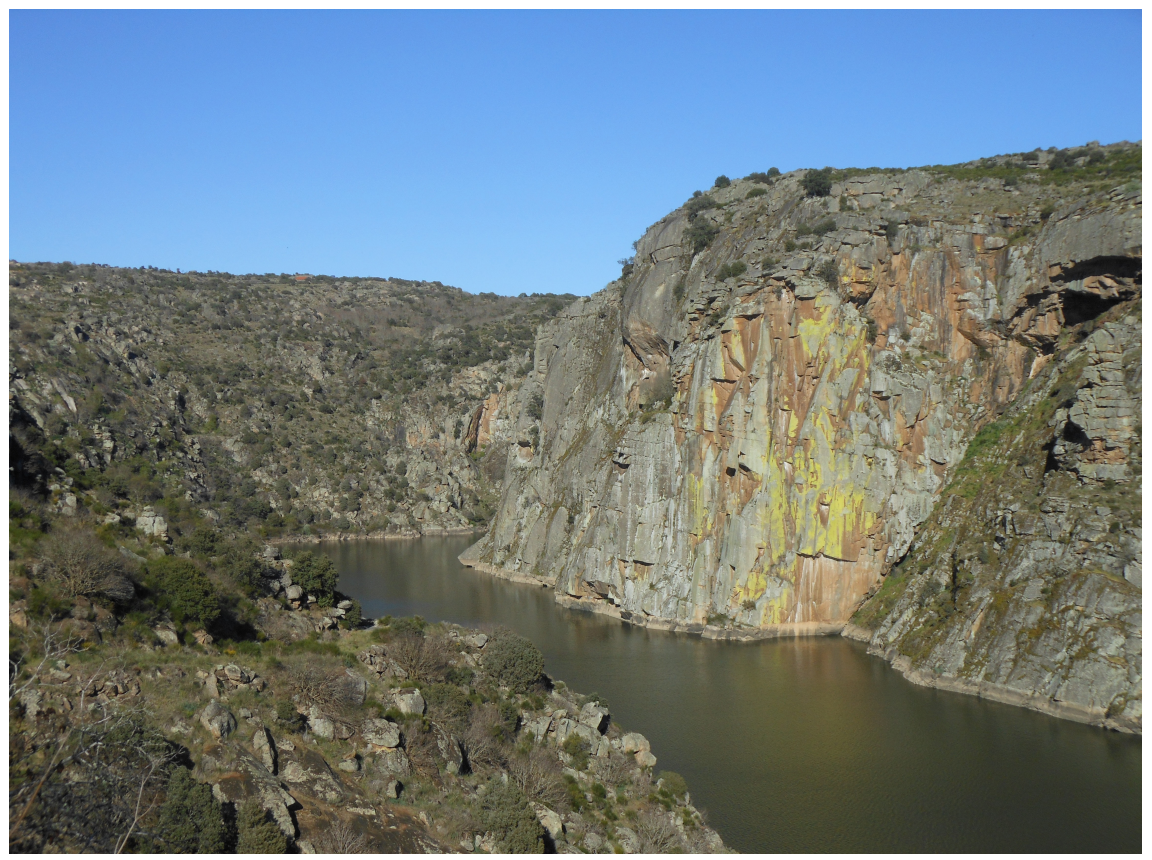

Fuente: fotografía de Valerià Paül (30 de marzo de 2015).

\section{Notas metodológicas}

La investigación se basa de manera preferente en el empleo de la técnica de las entrevistas semiestructuradas. Con ellas, se busca dejar hablar a los informantes con libertad, de modo que vayan fluyendo las ideas «que deberían permitir al investigador revelar las lógicas complejas de la acción puestas en marcha en los territorios» (Di Méo y Buléon, 2005: 37) ${ }^{5}$. Al mismo tiempo, gracias a una serie de temas que se presentan en las entrevistas, se persigue cubrir todos los aspectos de interés en la investigación, con el fin de que todas ellas resulten comparables (Ruiz Olabuénaga, 1999; Winchester y Rofe, 2010). Es por este motivo que se utiliza un guion de entrevistas, siguiendo las convenciones de autores como Valentin (1997) o Kitchin y Tate (2000). Se plantearon diversos bloques, tales como:

a) Descripción breve y personal del territorio, de forma espontánea, con el propósito de identificar representaciones asociadas.

b) Percepción personal sobre la zona y su evolución temporal.

5. Todas las traducciones al español de las citas de textos en lengua extranjera son propias. 
c) Detección de diversas personas o instituciones que operan en el territorio, para poder identificar actores y redes.

d) Percepción individual del rol jugado por el sector público en el desarrollo y ordenación del territorio.

e) Situación fronteriza del territorio, para encontrar áreas plausibles de cooperación, o barreras que lo impidan.

Estos temas se fueron planteando con alrededor de 30 preguntas sencillas, utilizando cuando era posible la fórmula "háblame de...» (Valentine, 2005), que facilita respuestas más abiertas. Cabe puntualizar que las técnicas de investigación cualitativa no buscan «respuestas objetivamente verdaderas, sino subjetivamente sinceras» (Ruiz Olabuénaga, 1999: 170), que permitan entender cómo la gente le da sentido a sus vidas (Valentine, 2005). En esta línea, es fundamental indicar que, de entrada, no se preguntaba de modo directo por la Reserva o por la cooperación entre países; de esta forma, se pretendía no prejuzgar o "contaminar» sus opiniones, y poder así observar cómo emergían estas realidades en la conversación. Para este estudio, se realizaron 13 entrevistas entre febrero y marzo de 2017, de una hora de duración aproximadamente. Los actores se han clasificado en cinco categorías: político local, técnico de la administración, gestor de conservación de la naturaleza, empresario y activista. Para garantizar el anonimato, las personas entrevistadas se mencionarán con un código alfabético - acompañado de uno de los descriptores cuando se nombran por primera vez, empleados en masculino con el propósito de no marcar el género-.

Las entrevistas, una vez solicitado el permiso de los informantes, fueron grabadas y transcritas. Su contenido fue posteriormente analizado a través de codificación abierta, lo que permite sintetizar la información obtenida de manera científica. Los códigos son tanto de tipo emic (extraídos de las palabras recogidas) o etic (establecidos por los investigadores), de acuerdo con Crang (1997) y Cope (2010). Estos códigos permiten elaborar un discurso de los resultados en el apartado que sigue. Se ha respetado la lengua original empleada por los interlocutores; se marcan en cursiva los elementos no normativos de las lenguas gallega, española o portuguesa, respectivamente.

\section{Resultados}

La cooperación en este territorio fronterizo parece sustentarse en la identificación de elementos compartidos a ambos lados de la raya. Se apuntan, por ejemplo, lazos comunes en cuanto a la cultura que «nos ha[n] permitido tener muchos proyectos en común» (MI/C, técnico de la administración), pues «[la] identificación transfronteriza a nivel cultural [es] tremenda, tremenda» (MI/B, técnico de la administración). Son también similares los déficits detectados. Así de claro se expresa MI/C cuando afirma que «como las necesidades eran las mismas, los problemas eran los mismos». O, en boca de MI/K (técnico de la administración): «os problemas deste lado da raia são os mesmos problemas 
do outro lado da raia». Unos inconvenientes que proceden de una situación marginal respecto al centro de poder de los respectivos estados:

É o poder central que ignora e acha que somos um parente pobre do resto do país e, por tanto, unindo forças dos dois lados da raia acho que fazemos mais força e temos mais presença, quer em termos nacionais (Portugal/Espanha), quer em termos europeus. (MI/K)

En este sentido, se menciona ZASNET como instrumento de coordinación de acciones conjuntas a ambos lados de la frontera. Un informante afirma que la figura de AECT para ZASNET es un paso adelante en la cooperación «porque hasta ahora no teníamos una entidad con personalidad jurídica» (MI/C). De este modo, se apunta que su valía fundamental es que «embora haja pontos de vista diferentes dos membros da assembleia que se sentam na mesa nas reuniōes, no final há sempre uma resolução» (MI/K). Sale a la palestra la identidad que se ha dado en generar entre ZASNET y la Reserva de la Biosfera: "ZASNET no se creó con la finalidad de la Reserva. Lo que pasa es que su proyecto estrella es la Reserva» (MI/B).

La amplia extensión de la Reserva parece haber estado en discusión desde sus inicios. En efecto, diversas personas informan de que en origen se proyectaba una extensión incluso mayor, pero que se tuvo que ir reduciendo porque «não era possível gerir um espaço tão grande» (MI/K). Parece incluso que se produjo oposición política al ámbito territorial escogido: «houve representantes da população que não quiseram entrar, de ai houve até recortes ainda maiores do que a última proposta que nós apresentamos» (MI/K). Aún así, el tamaño actual parece «abarcar demasiado», lo que conforma «una de las mayores reservas de la biosfera» (MI/F, empresario). En este mismo sentido, otro entrevistado la define como «una cosa un poco grande» (MI/D, gestor de la naturaleza), en la que se superponen distintas figuras de protección:

Aquello es LIC, que ahora es ZEC [...] es ZEPA [Zona de Especial Protección para las Aves] y ahora también la... Meseta Ibérica, que es una reserva de la biosfera muy grande que se ha hecho entre Portugal, Salamanca y Zamora. $(\mathrm{MI} / \mathrm{D})$

Otros informantes la mencionan, pero parecen no tener claros sus límites. Así, MI/J (técnico de la administración), que desarrolla su vida profesional en una zona incluida en la Reserva, expresa que "é mais para a zona de Miranda, na outra parte», como si no formara parte de la misma. Y MI/B no es capaz de localizar uno de los extremos de la Reserva, Pena Trevinca: «Pero no sé qué tiene que ver con... nosotros. Te lo digo porque la Reserva no llega ahí... [silencio]. No [...]. Es que no tenemos nada que hacer ni ver ahí».

En relación con los significados que se le asocian a la Reserva, de entrada, sorprende uno, que sería su intranscendencia. Por ejemplo, un entrevistado no la menciona al hablar de diversas figuras de protección; al preguntarle directamente por ella, comenta: «creo que es significativo que no me acuerde de la 
Reserva de la Biosfera; como si no existiera, vaya» (MI/I, activista cultural y naturalista). Esta percepción contrasta diametralmente con el miedo a que la Reserva conlleve limitaciones, lectura que emerge en varios informantes:

A zona LIC implica unha serie de... restricciois... Estamos tamén na Reserva da Biosfera [...]. En principio non hai restricciois como tal, pero si hai unha burocracia. (MI/H, político local)

Por el propio nombre, «Reserva de la Biosfera», iparece que te van a prohibir todavía más! (MI/E, político local)

Cuando se habló que toda la comarca iba a forma parte de la Reserva de la Biosfera se [le] pusieron los pelos de punta a la gente. (MI/G, gestor de conservación de la naturaleza)

No obstante, un contra-discurso a estas opiniones adquiere notoriedad cuando una serie de personas coinciden en afirmar que la Reserva no tendrá consecuencias en términos de protección o conservación de la naturaleza:

No implica legislación, ni se crea una figura nueva. La palabra «reserva» en sí da lugar a malas interpretaciones, y la gente se cree que la Reserva es un nuevo parque y [que] es una nueva legislación. (MI/B)

Son zonas que non son como os parques naturais, que ten probibiciois. Non te vai $a$ prohibir nada. Vai $a$ ser todo naturaleza... Bueno, naturaleza e turismo, é o que queren. [...] É como un sello de calidad! (MI/A, político local)

E incide:

A Reserva de la Biosfera [...] vaiche $a$ dare un sello, non che vai a dare subvenciois así en diñeiro, como... pra que tu poidas invertir. [...] Va a ser máis publicidad [...] que o que son ayudas económicas. (MI/A, político local)

Nótese cómo en esta última cita, y de modo paralelo a manifestar que la Reserva no implica conservación ni gestión en términos homologables a los de un espacio natural protegido, emerge la asociación de la misma con el turismo y la marca o sello. Este es, de hecho, un relato muy extendido, lo que puede estar evidenciando una suerte de «discurso oficial» que parece haber calado en buena parte de los entrevistados.

La Reserva de la Biosfera, al fin y al cabo, es un título. Es un marchamo de calidad. (MI/B)

Estamos tamén na Reserva da Biosfera, podemos disfrutar da «marca da biosfera». (MI/H)

Es una marca a mayores, que te van a dar gratis: ;toma, que estás dentro de esto! ¡Todo lo que te den gratis es bueno! (MI/G) 
En una de las entrevistas se recoge de qué modo se está difundiendo dicho «discurso oficial»; en una segunda, se advierten las dudas que han surgido al respecto:

Según eles [la Diputación] te beneficia, non te lastra. Según eles, que te venden a marca da Reserva. Esta iniciativa da marca levoua a cabo a Diputación de Zamora [...]. É unha ferramenta para potenciar incluso máis os recursos tipo turismo. $(\mathrm{MI} / \mathrm{H})$

Vino [alude a una persona vinculada a la Reserva] y hubo un poco de confusión, y nadie entendía muy bien para qué servía, si había algún tipo de mapas, a dónde llegaban con sus límites... Pero hablas con los alcaldes de la zona, de los ayuntamientos, y es igual. Que no hay información, que la Reserva de la Biosfera es algo vacío, una etiqueta general. (MI/I)

En lo que se refiere al turismo, parece no buscarse cualquier demanda, sino "turismo de natureza, turismo de aventura", y no "un turismo de massa" $(\mathrm{MI} / \mathrm{K})$. Los potenciales clientes, se presume, deberían llegar desde los centros urbanos, quizás espoleados por el hecho de que la Reserva podrá «ser la primera del mundo que tenga parada de AVE» (MI/B):

Acho que o potencial de turistas deste destino está nas grandes metrópoles da Península Ibérica e em países do norte da Europa. E acho que em nenhum destes pontos [...] há informação sobre este território, e acho que deveria haver. $(\mathrm{MI} / \mathrm{K})$

Este último entrevistado apunta a que la Reserva, cuyo valor promocional turístico se presume central, no está siendo suficientemente difundida de forma externa. Ello parece estar asociado con la ausencia de fondos, ya que «las ideas hay que calzarlas, porque si no... ¡calzarlas con pasta, con dinero!» (MI/E). En este sentido, la Reserva también se entiende como una suerte de lobby de presión para obtener fondos:

Y ahí han creado un lobby formal entre España y Portugal, para recibir dinero, los pacotes de investimiento para la Reserva de la Biosfera. Claro, normal. Que hagan algo. Por lo menos eso. (MI/L, empresario)

En este sentido, parece depositarse una cierta esperanza en que el mencionado sello sea una puerta para la obtención de recursos económicos: «tenemos que buscar una financiación [...] Estamos en ese camino» (MI/B). El valor de marca de la Reserva se supone que funcionará per se y se consolidará con el tiempo. Ocurriría lo mismo que en el caso de los parques naturales, desconocidos años atrás, y que, en la actualidad, se presentan como elementos social (y económicamente) asumidos:

La Reserva de la Biosfera, a largo plazo será positivo, y será como ahora mismo el Parque Natural del Lago de Sanabria [...] Cuando empezó a funcionar al 
principio probablemente no se comiesen una rosca o se comiesen pocas. Según ha ido avanzando, según ha ido pasando el tiempo, con el Lago de Sanabria, eh... llegamos a muchos más oídos. Ya lo conoce muchísima más gente. Con la Reserva pues llegará a pasar lo mismo. (MI/F)

En lo relativo al funcionamiento de la Reserva, se explica de qué modo se coordinan los equipos español y portugués:

[Las relaciones son] perfeitas. Sem problemas, as nossas reuniões são bilingues. Intentamos nosotras hablar español, pero... mas falamos português e elas entendem-nos. Leem os nossos documentos em português perfeitamente. Nós lemos os documentos deles em espanhol perfeitamente. (MI/K)

Estamos en constante comunicación, intercambiamos datos y ellas van a ferias, nos informan, nosotros tenemos [hemos] ido a una, y demás... Pero, bueno, cada uno hace su trabajo en comunicación constante. [...] Nosotros funcionamos en conjunto: entonces, las decisiones se toman en conjunto. Si no se decide en una asamblea, se hace en dos o tres. Hasta que salga la decisión. [...] Aquí no hay separatismos, no hay... que uno haga una cosa y Portugal no se entere. No, no. Las decisiones son... ¡ jen común! En una asamblea general común. (MI/B)

A pesar de las buenas relaciones personales y comunicativas aparentes, emerge en las entrevistas un problema de cariz administrativo. Y la raíz del mismo está en la pertenencia a dos estados-nación diferentes:

La mayor diferencia que yo puedo ver es la organización territorial de España: es distinta a la organización territorial de Portugal. Y a nivel legislativo, aunque hay siempre una base lógicamente común, pues bueno, cambian las nomenclaturas, cambian las formas de tramitar determinadas cuestiones... (MI/B)

\section{Discusión y conclusiones}

Las entrevistas realizadas apuntan hacia una visión particular de la Reserva de la Biosfera como ENPT. A pesar de que existen entrevistados que muestran un cierto temor a que el dispositivo implique restricciones en un sentido protector o conservador, emerge un "discurso oficial», difundido a través de reuniones explicitadas en las entrevistas, en el que se tiende a minusvalorar esa dimensión de la Reserva. Dicho discurso, en cambio, sustenta la idea de que la Reserva debería permitir "comerse una rosca» (MI/F). Esto es, que ha de servir sobre todo como sello, marchamo, marca o etiqueta del territorio - las cuatro son expresiones que aparecen en su literalidad en boca de los entrevistados-.

El ENPT pierde, de este modo, su sentido esperable, de manera que transita hacia el mundo del marketing y del turismo. Este resultado podría aparecer en el marco analítico como una posible derivada de todo el proceso, pero no como el vector, impulso o valor principal de la cooperación. No es sorprendente, en este sentido, que se recojan alusiones a la Reserva tales como algo 
«vacío» o «inexistente». Esto puede estar evidenciando que la Reserva se haya construido buscando un público exterior — es reveladora la mención al AVEy no se haya conformado desde el propio territorio en una lógica «de abajo arriba», lo que contradice las buenas prácticas (Braack et al., 2006; Leibenath et al., 2010; Erg et al., 2012).

Resulta interesante comprobar cómo muchos de los aspectos esperables en un ENPT de acuerdo con la bibliografía de referencia ni siquiera se mencionan por parte de los entrevistados: normativa, plan de acción, monitoreo, desarrollo territorial vinculado a la naturaleza, etc. (Sandwith et al., 2001; Braack et al., 2006; Lange, 2009; McKinney y Vasilijević, 2012). Esto no niega su existencia, sino que remarca su desconocimiento o su irrelevancia para los entrevistados, lo que es significativo de por sí. Ciertamente, se mencionan estructuras de cooperación entre los dos lados de la frontera — facilitadas por la intercomprensión de las lenguas peninsulares—, algo esperable según Büscher y Schoon (2009) u Oszlanyi (2001). Sin embargo, la AECT no parece aquí ser asimilable, como en los casos franco-italianos, a un ENPT. En relación con la cuestión financiera, las menciones a que la Reserva debería generar ingresos de forma directa o indirecta (a través de la marca, el sello, la promoción, etc.) adquieren relevancia, lo que confirma autores como Lange (2009), Büscher (2010), Leibenath et al. (2010) o Trillo y Paül (2016) cuando aluden a que las estructuras de cooperación transfronteriza parecen correlacionarse con la captación de recursos económicos.

Por todo lo anterior, el carácter transfronterizo de la Reserva puede haber funcionado como una suerte de pretexto con el fin de poder alcanzar su declaración. Se evidencia, además, una cierta obsesión por conseguir unas dimensiones tales que la conviertan en la más grande de la Península Ibérica, lo que puede ser leído, una vez más, en clave de marketing. De este modo, la Reserva parece alejarse de la voluntad de proteger, conservar o gestionar la naturaleza. Tampoco se infiere que proceda de un proceso participativo de calado que se fundamente en las opiniones de las personas que viven en ese ámbito. Este hecho está en contra del parecer de instituciones y expertos internacionales en cooperación transfronteriza, que proclaman la necesidad de asegurar una genuina participación ciudadana en la gobernanza transfronteriza para que pueda ser exitosa (Bianco y Bianchizza, 2015; Comisión Europa, 2017). Si esto no es así, corremos el riesgo de perpetuar los proyectos de regiones transfronterizas como «regiones en los discursos» $\mathrm{o}$ "regiones en el papel», pero no como parte de las prácticas sociales (Paasi, 2002: 200).

\section{Referencias bibliográficas}

Bianco, Daniele del y Blanchizza, C. (2015). European experience of citizen's participation in cross-border governance. Gorizia: ISIG/Council of Europe.

Braack, Leo; Sandwith, Trevor; Peddle, David y Petermann, Thomas (2006). Security considerations in the planning and management of transboundary conservation areas. Gland/Cambridge: International Union for Conservation of Nature. 
BÜSCHER, Bram (2010). «Seeking 'telos' in the 'transfrontier'? Neoliberalism and the transcending of community conservation in Southern Africa». Environment and Planning A, 42, 644-660. $<$ https://doi.org/10.1068/a42140>

BÜSCHER, Bram y SCHOON, Michael (2009). «Competition over conservation: collective action and negotiating transfrontier conservation in Southern Africa». J. Int. Wildl. Law Policy, 12 (1), 33-59. <https://doi.org/10.1080/13880290902938138>

Comisión Europea (2017). Comunicación de la Comisión al Consejo y al Parlamento Europeo. Impulsar el crecimiento y la cohesión en las regiones fronterizas de la UE. COM(2017) 534 final.

Cope, Meghan (2010). "Coding Qualitative Data». En: HAY, I. (ed.). Qualitative research methods in human geography. Don Mills: Oxford University Press, 281-294.

CRANG, Mike (1997). "Analyzing qualitative materials». En: FlOWERDEW, R. y MARTIN, D. (eds.). Methods in human geography. Harlow: Longman, 183-196.

Depraz, Samuel (2008). Géographie des espaces naturels protégés. Genèse, principes etenjeux territoriaux. París: Armand Colin.

ERG, Boris; VASILIJEVIĆ, Maja y MCKINNEY, Matthew (eds.) (2012). Initiating effective transboundary conservation. A practitioner's guideline based on the experience from the Dinaric Arc. Gland/Belgrade: International Union for Conservation of Nature.

EUROPARC Federation (ed.) (2010). Following nature's design. Promoting cross-border cooperation in nature conservation. Regensburg: EUROPARC Federation.

KITCHIN, Rob y TATE, Nick J. (2000). Conducting research into human geography. Theory, methodology and practice. Harlow: Prentice Hall.

LANGE, Sigrun (2009). Transboundary cooperation in protected area's management factors for success or failure. Klagenfurt: University of Klagenfurt. [Tesis de máster]

LEIBENATH, Markus; Blum, Andreas y STUTZRIEMER, Sylke (2010). «Transboundary cooperation in establishing ecological networks: the case of Germany's external borders». Landscape and Urban Planning, 94, 84-93. $<$ https://doi.org/10.1016/j.landurbplan.2009.08.002>

LÓPEZ TRIGAL, Lorenzo (2017). «Investigación geográfica sobre las fronteras de la península Ibérica». Polígonos, 29, 327-346. <https://doi.org/10.18002/pol.v0i29.5213>

LÓPEZ Trigal, Lorenzo et al. (1995). La cooperación de Castilla y León con Portugal. Relaciones transfronterizas. Valladolid: Cortes de Castilla y León.

LÓPEZ TRIGAL, Lorenzo y GUICHARD, François (coords.) (2000). La frontera hispanoportuguesa: nuevo espacio de atracción y cooperación. Zamora: Fundación Rei Afonso Henriques.

Méo, Guy Di y BulÉon, Pascual (2005). L'espace social. Lecture géographique des sociétés. París: Armand Colin.

MCKInNeY, Matthew y VASILIJEVIĆ, Maja (2012). "Guidelines for initiating transboundary conservation». En: ERG, Boris; VASILIJEVIć, Maja y MCKINNEY, Matthew (eds.). Initiating effective transboundary conservation. A practitioner's guideline based on the experience from the Dinaric Arc. Gland/Belgrade: International Union for Conservation of Nature, 24-41.

OsZLANYI, Julius (2001). Report of the Ad hoc task force on transboundary biosphere reserves. UNESCO. Seville +5 . International Meeting of Experts. Proceedings = Comptes rendus = Actas. Pamplona, Spain 23-27 October 2000: United Nations Educational, Scientific and Cultural Organization, 55-57. 
PAASI, Anssi (2002). "Regional transformation in the European context: notes on regions, boundaries and identity». Space Polity, 6 (2), 197-201. <https://doi.org/10.1080/1356257022000003626>

PaÜl, Valerià; CASTAÑer, Margarida; Trillo, Juan M.; Martín, Javier y ViCente, Joan (2017). "La participación española en la cooperación transfronteriza y territorial europea». En: FARINÓs, Joaquín (ed.). Geografía regional de España: espacios y sociedades. Valencia: Tirant lo Blanch, 617-675.

PRADA, Esther Isabel (2014). "Sayago: "paisaje fuente”, o la construcción del lugar en la frontera hispano-portuguesa». En: Molinero, Fernando (coord. gral.). Atlas de los paisajes agrarios de España. Madrid: Ministerio de Agricultura, Alimentación y Medio Ambiente, II, 1021-1032.

Ruiz Olabuénaga, José I. (1999). Metodología de la investigación cualitativa. Bilbao: Universidad de Deusto.

SANDWITH, Trevor y LOCKWOOD, Michael (2006). "Linking the landscape». En: LOCKWOOD, Michael; Worboys, Graeme y Kothari, Ashish (eds.). Managing protected areas. A global guide. Londres/Sterling: Earthscan, 574-602.

SANDWITH, Trevor; SHINE, Clare; Hamilton, Lawrence y SHEPPARD, David (2001). Transboundary protected areas for peace and cooperation. Gland/Cambridge: International Union for Conservation of Nature.

TRILlO, Juan M. y PAÜL, Valerià (2016). «Transboundary protected areas as ideal tools? Analyzing the Gerês-Xurés transboundary biosphere reserve». Land Use Policy, 52, 454-463. <https://doi.org/10.1016/j.landusepol.2015.12.019>

VALENTINE, Gill (2005). «Tell me about...: using interviews as a research methodology». En: Flowerdew, Robin y MARTIN, David (eds.). Methods in Human Geography. Harlow: Longman, 110-126.

WinCHESTER, Hillary P. M. y Rofe, Matthew W. (2010). "Qualitative research and its place in human geography». En: HAY, Iain (ed.). Qualitative research methods in human geography. Don Mills: Oxford University Press, 3-25. 